\title{
Effect of Medicine on Interleukin-6 Inhibitors and their Forms Against COVID-19
}

\author{
Kevser Kusat ${ }^{1}$ (D) , Sinan Akgöl $2, *$ (i) \\ 1 Turkish Medicines and Medical Devices Agency Ministry of Health, Ankara, Turkey; kkusat@hotmail.com (K.K.); \\ 2 Ege University Faculty of Science Biochemistry Department, Bornova, Izmir, Turkey; sinanakgol@gmail.com (S.A.); \\ * Correspondence: sinanakgol@gmail.com (S.A.);
}

Scopus Author ID 6603594798

Received: 24.02.2021; Revised: 15.04.2021; Accepted: 18.04.2021; Published: 26.04.2021

\begin{abstract}
Coronavirus disease-2019 (COVID-19) is caused by severe acute respiratory syndrome coronavirus 2 (SARS-CoV2), previously known as the 2019 novel coronavirus (2019-nCoV). The first cases were seen in December 2019 in Wuhan. To date, there is no specific treatment for COVID-19 disease. Recent clinical trials suggest that interleukin-6 (IL-6) inhibitors and their derivates could play a role as a therapeutic agent against COVID-19. It is prominent to assess potential therapeutic effects of drugs that are clinically approved for other indications. In addition, IL-6 inhibitors have been used in nanomedicine. This review focuses on the development of nanosystems for the therapy of COVID19. IL-6 inhibitors and signal pathway inhibitors attached to biocompatible nanomaterials may provide a useful route for the treatment of COVID-19. This review would also help scientists who make potential drug research via attachment of interleukin-6 inhibitors and their derived forms to biocompatible nanomaterials for treatment of COVID-19.
\end{abstract}

Keywords: COVID-19; interleukin-6; IL-6/JAK/STAT3 signal pathway inhibitors; nanomedicine; nanotechnology; theranostic strategies

(C) 2021 by the authors. This article is an open-access article distributed under the terms and conditions of the Creative Commons Attribution (CC BY) license (https://creativecommons.org/licenses/by/4.0/).

\section{Introduction}

According to the World Health Organization (WHO), viral diseases continue to emerge and display serious public health issues. Various viral epidemic diseases such as severe acute respiratory syndrome coronavirus (SARS-CoV) in 2002 to 2003, H1N1 influenza in 2009, and Middle East respiratory syndrome coronavirus (MERS-CoV) in 2012 have been recorded for the last twenty years [1]. Novel coronavirus turned out in Wuhan (Hubei, China) in December 2019 and spread rapidly worldwide. Various coronaviruses may lead to light respiratory disease. Nevertheless, SARS-CoV-2 could lead to pneumonia, called coronavirus disease-2019 (COVID-19) that is the leading cause of death because of massive alveolar damage and progressive respiratory failure [2]. WHO announced the COVID-19 pandemic on March 12, 2020 [3].

Coronavirus is enveloped, nonsegmented, positive-sense single-stranded RNA virus genomes in size 26-32 kilobases. The family Coronaviridae, which comprises alpha, beta, delta, and gamma coronaviruses. The virus has nucleocapsid formed of genomic RNA and phosphorylated nucleocapsid $(\mathrm{N})$ and covered by spike proteins $(\mathrm{S})$. The membrane $(\mathrm{M})$, which is a type III transmembrane glycoprotein, and envelope $(\mathrm{E})$ protein are located among $\mathrm{S}$ proteins in the virus envelope [4, 5, 37], as you can see in Figure 1. 

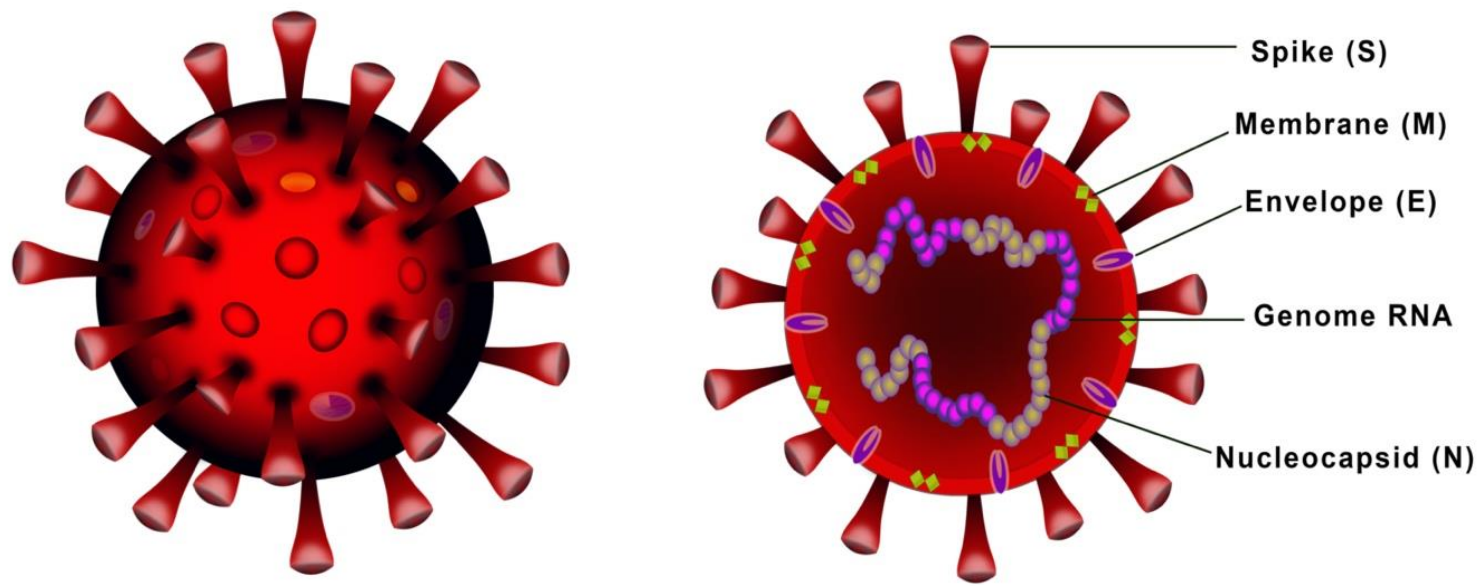

Figure 1. Structure of coronavirus.

Coronavirus spike protein (S) mediates entry into target cells by binding to the cellular receptor and fusing the viral envelope with the host cell membrane. $\mathrm{S}$ forms two functional subunits: S1 subunit, responsible for binding to host cell receptor, S2 subunit; the fusion of viral and cellular membranes. S protein is cleaved at the boundary between $\mathrm{S} 1$ and $\mathrm{S} 2$ subunits, which remain non-covalently bound in a prefusion conformational state. This cleavage is proposed to activate protein for membrane fusion by means of irreversible conformational modifications. Therefore, coronavirus enters into the cells. The complex process needs the compatible action of receptor-binding and proteolytic processing of S protein to promote viruscell fusion [6-8].

Figure 2 presents the summary for the life cycle of COVID-19. S protein uses angiotensin-converting enzyme 2 (ACE-2) is a receptor for host cell entry. ACE-2, a metallopeptidase, is expressed on major viral target cells, type II pneumocytes, enterocytes. Its catalytic domain binds to $\mathrm{S}$ protein with high affinity. The binding of $\mathrm{S}$ protein to ACE-2 triggers subtle conformational changes from this protein. These conformational modifications are rise sensitivity of $\mathrm{S}$ protein to proteolytic digest at the border between $\mathrm{S} 1$ and $\mathrm{S} 2$ subunits $[9,11]$. The S protein engages ACE-2 as the entry receptor and employs cellular serine protease TMPRSS 2 for S protein priming. The efficiency of ACE-2 usage was found to be a key marker of coronavirus transmissibility [10].

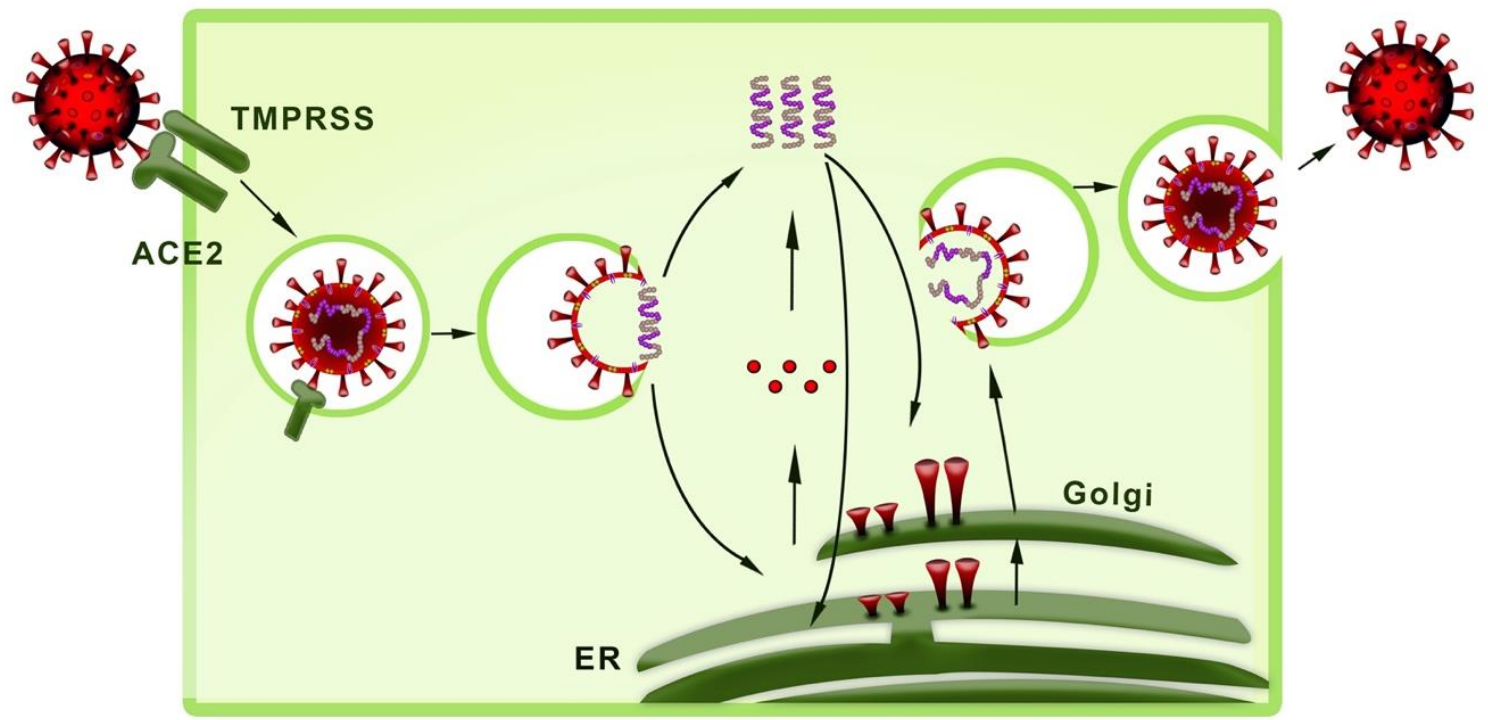

Figure 2. The life cycle of COVID-19. 
COVID-19 can lead to serious damage to the respiratory tract, involving the lung. COVID-19 may result in mild or highly acute respiratory syndrome with the resulting release of pro-inflammatory cytokines, involving IL-1 $\beta$ and IL-6. Binding of COVID-19 to Toll-Like Receptor (TLR) causes of pro-IL-1 $\beta$ release. Pro-IL-1 $\beta$ is cleaved by caspase-1, followed by inflammasome activation as well as the formation of mature IL-1 $\beta$. The active IL- $1 \beta$ is the mediator of lung inflammation, fever, and fibrosis. Inhibition of IL-1 and IL-6 has been revealed a therapeutic effect in many types of inflammatory diseases, including viral infections [12].

Inflammation is a natural defense mechanism that includes the migration of leucocytes to damaged tissues. Infectious and allergic reactions stimulate the initial phase of acute inflammation. However, continued acute inflammation causes chronic inflammation. Acute inflammation is characterized by infiltration of neutrophilic cells followed by monocytic cells, while chronic inflammation is characterized by the presence of mononuclear cells such as macrophages and lymphocytes in the inflammation area. Moreover, several cytokines are produced during inflammation. These act in synergy and have an overlapping activity that can happen both receptor and along intracellular signaling pathways. The cytokines mainly involve in IL-6, IL- $1 \beta$, TNF- $\alpha$, IFN- $\gamma$, and TGF- $\beta$, which induce the production of acute-phase proteins. IL-6 plays a crucial role in both reveals acute phase reactions and causes cellular and humoral immune responses. Therefore, IL-6 is a notable modulator for the transition from the acute phase to the chronic phase of the inflammatory process [13, 14, 17, 37].

\section{Signal Mechanisms of IL-6}

IL-6 was cloned as a B-cell stimulatory factor by Kishimoto and coworkers in 1986 (16). IL-6 is 184 amino acid glycosylated protein, which can be synthesized and secreted by many cell types involving monocytes, T-cells, fibroblasts, and endothelial cells. IL-6 binds to a specific membrane receptor (IL-6R), which is an $80 \mathrm{kDa}$ type I transmembrane protein. IL-6 bound to IL-6R associates with a second transmembrane protein, gp130, which serves as a signal transducer of IL-6 (15). IL-6 is a multifunctional cytokine that plays a role in both the pro-inflammatory and anti-inflammatory processes $[4,17]$.

\subsection{IL-6 receptor system and signal pathways.}

IL-6 signaling is mediated by two different pathways: classic and trans-signaling pathways. Classic signaling includes binding of IL-6 to IL-6R on the cell surface and following interaction of this complex with gp130 to start intracellular signaling. In the trans-signaling pathway, IL-6 binds to secreted form of IL-6R (sIL-6R), followed by the interaction of this complex with gp130. Each pathway regulates different biological effects. A classic signaling pathway is especially substantial for acute-phase immunological response, homeostasis etc. The trans-signaling pathway has a crucial role in the tumor microenvironment, etc. [18].

IL-6 binds to IL-6R and associated IL-6/IL-6R complex, stimulating homodimerization of gp130. IL-6-related cytokine receptors induce intracellular signals through Janus kinasesignal transducer and activator of transcription pathway (JAK-STAT), where receptorassociated JAKs (JAK1, JAK2, and TYK2) stimulates tyrosine phosphorylation of itself and activate the latent transcription factors STAT1, STAT3. Other signaling intermediates activated in response to IL-6 include, first, tyrosine phosphatase SH-PTP2 (SHP2; also known as PTPN11), which promotes activation of RAS-RAF-extracellular-signal regulated kinase1 
(ERK1)/ERK2 mitogen-activated protein kinase (MAPK) and phosphoinositide3-kinase (PI3K)-protein kinase B (AKT) pathways, and, second, transcription factor nuclear factor NFIL-6 (C/EBP $\beta)[14,19,20]$ as you can see in Figure 3.

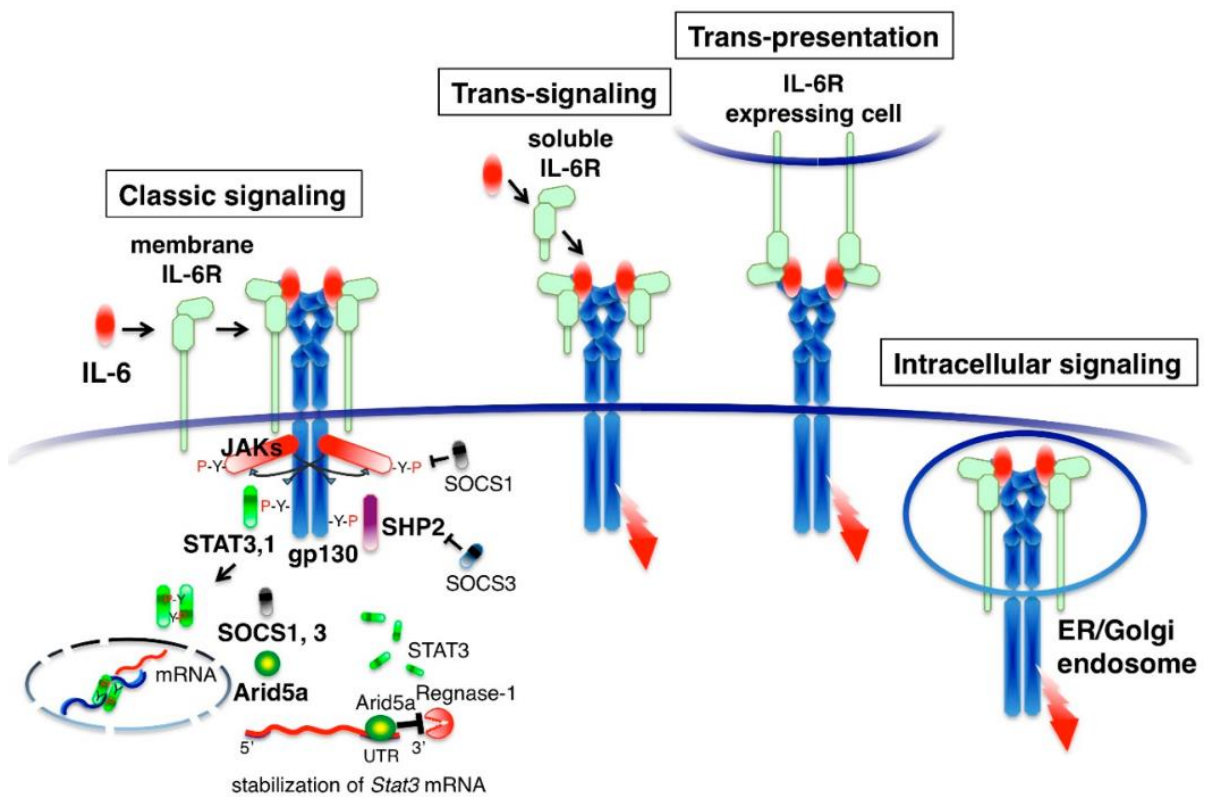

Figure 3. Signal mechanisms of IL-6. IL-6 signaling is mediated by two different pathways: classic and transsignaling pathways [19].

IL-6 contributes to specific physiological and immunological responses such as; autoimmune disease, cardiovascular disease, cancer, Alzheimer's disease, etc. [14, 17, 18, 20].

\subsection{IL-6 and signal pathway associated inhibitors.}

\subsubsection{IL-6 inhibitors.}

Tocilizumab, a humanized monoclonal antibody against IL-6 receptor, was approved by US Food and Drug Administration (FDA) and by European Medicines Agency. Tocilizumab was approved in 2005 based on its effectiveness in Castleman disease in Japan $[14,21]$. (Figure 5).

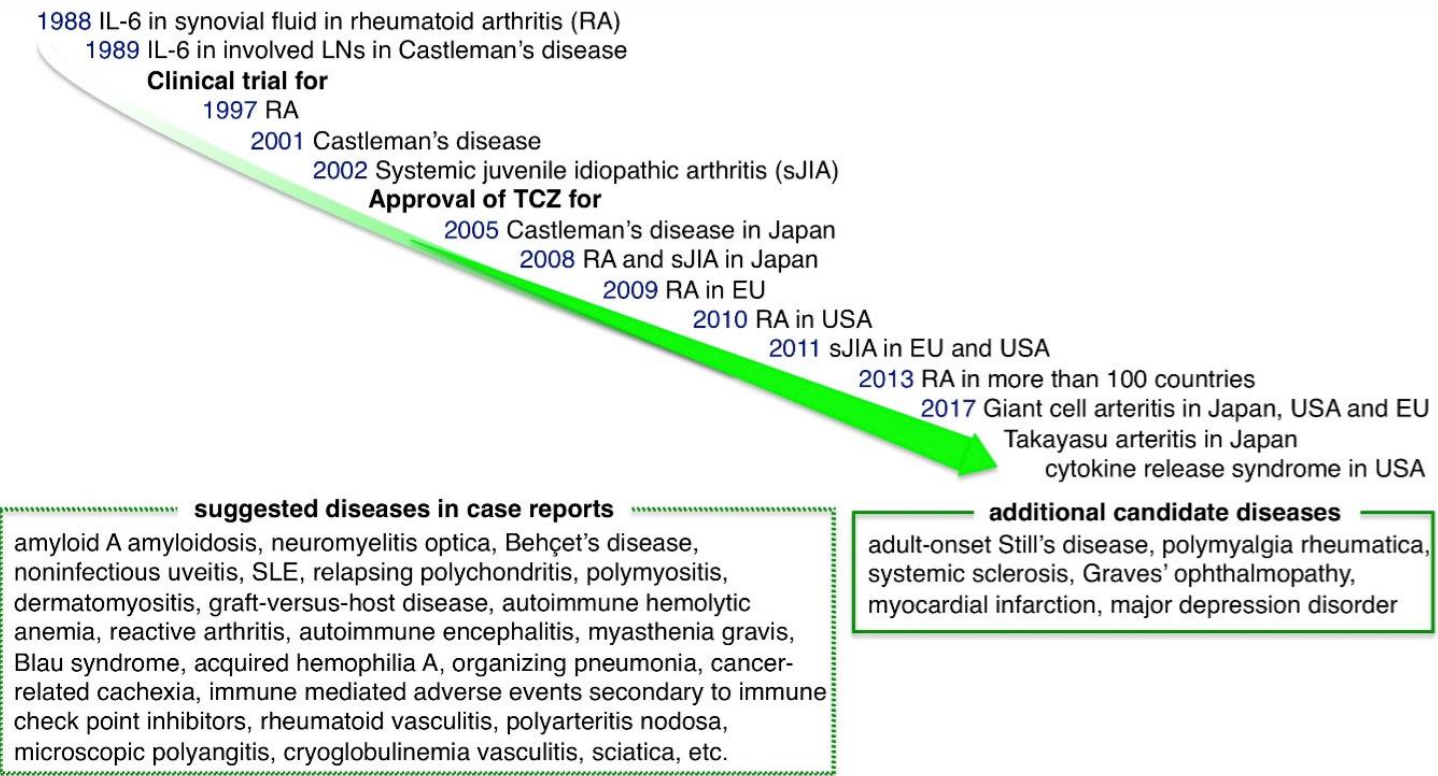

Figure 4. İnterleukin 6 inhibition treatment of various diseases [19]. 
Tocilizumab is used to treat Castleman disease, rheumatoid arthritis (RA), systemic and polyarticular juvenile idiopathic arthritis, giant cell arteritis, Takayasu arteritis, and cytokine releasing syndrome. Many case reports have shown that blocking IL-6 effectively treats many diseases $[19,54]$.

Similarly, Siltuximab is also an antibody that targets IL-6 and the IL-6 receptor- $\alpha$, was approved by the FDA to treat multicentric Castleman disease [18]. Clinical studies on anti-IL6 monoclonal antibodies such as sirukumab, olokizumab, elsilimomab, clazakizumab, sarilumab are in different phases of studies to set up their efficacy and safety in disease states. Various studies have revealed that monoclonal antibodies are beneficial therapeutic effects in IL-6-mediated diseases [14].

\subsubsection{IL-6/JAK/STAT3 signal pathway inhibitors.}

IL-6/JAK/STAT3 signaling pathway contributes to a variety of diseases. Strategies to inhibit this pathway involve inhibition IL-6/IL-6R complex, inhibition of JAK activation, inhibition of STAT3 phosphorylation, and inhibition of STAT3 DNA-binding activity [14].

\subsubsection{Jak inhibitors.}

Tofacitinib, ruxolitinib, and pacritinib have still been the most widely researched inhibitors of JAKs presently in preclinical improvement. Tofacitinib is a small-molecule tyrosine kinase inhibitor that particularly targets JAK1 and JAK3 and is approved by the FDA for use in arthritis, while ruxolitinib is a small-molecule inhibitor of JAK1 and JAK2 and was approved for the treatment of myelofibrosis and polycythemia vera [18]. In addition, filgotinib was identified as a selective inhibitor for JAK1 and JAK2 in cellular in vitro signaling assays. Filgotinib is presently in phase III trials for Crohn's disease, RA, and ulcerative colitis. Another JAK1-selective inhibitor, upadacitinib, has been tested in phase II trials for RA. Similarly, PF04965842, JAK 1-selective inhibitor, in phase II trials for atopic dermatitis is completed, yet unpublished [22].

\subsubsection{STAT 3 inhibitors.}

Tyrosine-phosphorylated peptides and peptidomimetics can bind to the $\mathrm{SH} 2$ domain of STAT3 and destroy STAT3 dimerization and DNA-binding activity [18]. Zinzalla et al. synthesized pyrrolidine-sulphonyl aryl molecules. Their studies indicate that the molecule is cytostatic and capable of selectively blocking STAT3 activation following IL-6 induction at both phosphorylation and transcriptional levels [23]. Singh et al. synthesized oxazolidine and indole derivatives and examined them as IL-6 signal pathway inhibition [24]. Guo et al. reported small molecules of benzensulfanilamide derivatives were prepared to inhibit STAT3 activation for anticancer treatment [25] selectively. Chrysin (5,7-dihydroxyflavone) can downregulate the expression of gp130, sIL-6R, phosphorylated JAK1, and STAT3. In addition, apigenin, luteolin, hispidulin, nepetin, and crisimaritin display inhibitory effect against STAT3 activity [14].

\section{Anti-inflammatory Treatment in COVID- 19}

The potential targeted monoclonal antibodies against IL-6 can selectively block this process and improve patients' clinical outcomes with evident features of cytokine-driven inflammation like persistent fever, dyspnea, and elevated inflammatory markers. 
When the virus enters the respiratory tract, it causes the release of cytokines such as interleukin IL-1 and IL-6. Releasing cytokines causes further lung inflammation, fever, and fibrosis. So, treatment of the cytokine storm has become an important part of rescuing severe COVID-19 patients. IL-6 plays an important role in cytokine release syndrome. Suppose it is possible to block the signal transduction pathway of IL-6. In that case, it is expected to become a new method for treating severe COVID-19 patients [50, 53].

Wei Haiming and coworkers reported that a retrospective study observing the effect of intravenously injected tocilizumab against severe or critical COVID-19 infection. Within a few days, the fever returned to normal and other symptoms improved significantly. The result suggests tocilizumab may be an effective therapeutic agent in severe COVID-19 pneumonia [26].

COVID-19 might invade and binding coronavirus S protein to ACE-2 receptors enter cells via endocytosis. One of the known regulators of endocytosis is AP2-associated proteinkinase1 (AAK1). AAK1 inhibitors may be a beneficial effect in preventing COVID-19. Baricitinib, JAK inhibitor, and AAK1 inhibitor were proposed as possible candidates for the treatment of COVID-19, considering its relative safety and high affinity [26, 27].

\section{From Diagnosis and Therapy to Theranostic Approaches for COVID-19}

The improvement of multifunctional materials and new strategies based on nanotechnology has generated new clinical challenges. An important challenge is the need to evaluate the treatment's long-term effect following changes in cells' cellular processes and physicochemical characteristics. Based on their intrinsic properties, nanomaterials such as nanocomposites, nanoconjugates, and others, with optical and magnetic properties, are used as contrast agents, devices for drug release. Different chemical interactions between drugs and functional polymers can be used in both diagnosis and treatment. The novel approaches gave an increase to the term "theranostics", which the National Institutes of Health describe (NIH) as the application of nanobiomaterials and devices at the molecular level for personalized diagnosis, imaging, and therapy [38, 39]. Theranostic strategies based on nanotechnology will start to be used for COVID-19 treatment in the near future.

\subsection{An important subject for COVID-19 treatment based on theranostics approach: toxicity of nanomaterials.}

Although nanomaterials' perfect potential for theranostic applications, it is important to consider their potential toxic effects. Although nanomaterials' nanotoxicological aspects are beyond the scope of this review, it is necessary to briefly consider the potential adverse effects of nanomaterials with theranostic applications. The potential risks of nanotechnology, which offer a significant place in many applications to humans, must be understood and researched. The biological activity and toxicity of nanomaterials depend on many factors such as size, surface chemistry, and other properties. There are several routes of these materials via oral, dermal, and inhalation, depending on use patterns. Exposure to nanomaterials may occur by any of these routes. When the nanomaterials enter the body, they can be distributed to various organs where they may remain structurally the same, become modified, or metabolized, such as metabolites. For more details on this topic, we refer readers to a published review [40]. Once nanomaterials inside the cells, they may cause several biological responses. For instance, dust particles can lead to several health problems by the inhalation route, especially in subjects with 
asthma patients. So, it is clear that it should be known how nanomaterials interact with biological systems. Hillyer and Albrecht observed that when metallic nanomaterials of 58, 28, 10 and $4 \mathrm{~nm}$ in size were orally administered to mice, biodistribution to other organs increased. The smallest particle ( $4 \mathrm{~nm}$ ) was the increased presence of gold particles in the kidney, liver, spleen, lungs, and even the brain. The biggest particle $(58 \mathrm{~nm})$ was detected almost only inside the gastrointestinal tract [41]. In another study, when intravenously injected gold nanorods revealed that within $30 \mathrm{~min}$, particles accumulated predominantly in the liver. Consequently, it should be known how nanomaterials interact with biological systems. Many health concerns have been present because of nanomaterials' exposure, including potential adverse effects on respiratory and cardiovascular systems, blood, gastrointestinal tract, skin, liver, and central nervous system. Blood is the first target in the case of intravenous administration of nanomaterials that gives body response information in medical diagnostics and therapy [42]. The use of nanomaterials contains some different potential risks in medicine. Some strategies are outlined to address some of these risks. Given nanomaterials' intrinsic properties, their safe clinical application would be different from the classical paradigm established for conventional drugs. In this regard, FDA and EU agencies have already established robust schemes to approve novel nanopharmaceuticals [43].

\subsection{Use of theranostic approach based on nanomaterials for COVID 19 treatment.}

The use of functional nanomaterials for diagnosis, prognosis, and treatment in medicine is developing rapidly in the last decade. Therefore, the functional nanomaterials are designed to use drug release and gene delivery systems, sensor applications, diagnosis, and prognosis for various diseases. These nanomaterials have the perfect potential for the therapeutic effect of different diseases [51, 52].

Otherwise, functional nanomaterials have gained great importance in the improvement of treatment and diagnostic equipment. Their physical, chemical properties could be modified with the easy adaptation of different biomolecules, such as targeted molecules, antibodies complementary to receptors particularly expressed on the target tissues [29, 30].

Furthermore, a combination of functional nanomaterials, such as natural nanomaterials like chitosan, alginate or biocompatible synthetic dendrimers, liposomes, inorganic nanomaterials, etc., gives hybrid nanomaterials with additional and extraordinary properties. These enhanced properties are acquired by attaching magnetic nanomaterials with polymeric matrix, or the more reliably protection of drug indicated by silica/polymeric nanomaterials than organic nanomaterials [31-35]. As we mentioned above, nanomedicines have been applied to treat and diagnose different diseases with promising results, but medical imaging and cancer disease are still the most active areas of improvement. Some examples of clinically approved intravenous nanomaterials are Doxil@(liposomal composition with doxorubicin for the treatment of various cancers) and Resovist ${ }^{\circledR}$ (iron carboxy dextran colloid as magnetic resonance imaging (MRI) contrast agent for imaging of liver lesions). Nanomaterials present in clinical trials are mainly aimed at treating several cancers [44]. One example relates to incorporating these materials into magnetic particles and directing the nanomaterials to target (e.g., cancer cells) by passing the magnetic fields formed around the cancer cell. This provides perfect advantages, such as decreased dosage and side effects and increased therapeutic effect [28]. Lee and coworkers prepared hyaluronate gold nanoparticle attached Tocilizumab (HA-AuNP/TCZ) nanomaterial to treat rheumatoid arthritis. Besides, 
they found that HA-AuNP/TCZ nanomaterial was very effective as a dual-targeting drug candidate to VEGF and IL-6R for treatment of RA [36].

Nanomaterials represent a novel strategy for preventing, diagnosing, and treating atherosclerosis and new multifunctional nanomaterials. Some studies show the role of different nanostructures in the search for better diagnostic and therapeutic approaches to tackle increasing cardiovascular diseases such as atherosclerosis and MI [45, 51, 55].

Gold nanomaterials that have antibodies attached could provide a quick diagnosis of the flu virus. When light is directed on the sample and the nanomaterials, the amount of light reflected back increases because nanomaterials cluster around virus particles, allowing a much faster test than those currently used [46].

One question may be useful for explaining the new route for COVID-19 treatment; Should we apply nano-therapy to interleukin-6 inhibitors and their derived forms against COVID-19? Firstly, nanomaterials' size impact on their in vivo behavior is one of the bestresearched aspects of nanomaterials pharmacokinetics and biodistribution. Presently, it is accepted that $10-100 \mathrm{~nm}$ is the optimal size for all medical purposes. Moreover, small particle size rises accumulation and enhances the penetration into tissue [47, 48].

Therefore, nanomaterials size and surface composition are substantial determinants to achieve effective COVID-19 therapy [56]. In addition, interleukin-6 inhibitors and/or their derived forms attached biocompatible nanomaterials dispersion and variation in size play a major role in their in vivo behavior. Polydispersed nanomaterials tend to possess a range of retention times and biodistribution. Thus, a controlled synthesis, ideally creating nanomaterials with identical properties in size, shape, charge, and, in the case of functionalization, amount of interleukin-6 inhibitors and/or derived forms bound to the nanomaterial's surface, should be sought if a uniform distribution is required. Another property of nanomaterials is their multivalency. Nanomaterials are characterized by a high surface area to volume ratio, causing high loading capacity for therapeutic formulations. For instance, a carbon nanotube with a similar volume to a typical large protein (100-150 kDa) offers a 15-fold larger surface area than the protein [49]. The enlarged surface area allows the coupling of many interleukin-6 inhibitors and/or derived forms against COVID-19 to the nanomaterials [52].

In the light of these informative data, we can say that interleukin-6 inhibitors and/or their derived forms attached to biocompatible nanomaterials can be used for COVID-19 treatment as a drug release system. There is required for improvement of novel nanointerleukin-6 inhibitors and derivates having low antigenicity and being patient-friendly. In conclusion, as we mentioned above, targeting IL-6/JAK/STAT3 signal pathway has been reported in the literature to be useful in treating many types of diseases. The improvement of rational combination monoclonal antibodies is required to synergistically target the IL6/JAK/STAT3 pathway and be used in the accurate treatment of COVID-19 infection.

\section{Conclusions And Perspectives}

Nanotechnology has had promising advances, such as different disease treatments. This review focuses on developing nanosystems for the treatment and diagnosis of COVID-19 and the evolution of nanosystems as theranostic tools. Development of theranostic strategies for COVID19 would be attracted much attention stimulating research with the potential to reach the clinic. Given all that has been mentioned so far, one may suppose that this review would also help scientists design, via novel nanomaterial attach of inhibition IL-6 via either mechanism. IL-6 inhibitors and/or signal pathway inhibitors may prove useful nano-drugs 
treatment of COVID-19. Some of these monoclonal antibodies have expensive, invasive routes and high antigenicity major limitations. Thus, there is a need to improve novel nano-IL-6 inhibitors and derivates with low antigenicity and being patient-friendly.

The relationship between anti-inflammatory treatment and infection disease has been widely investigated. In conclusion, targeting IL-6/JAK/STAT3 signal pathway has been reported in the literature to be useful in treating many types of diseases. The improvement of rational combination monoclonal antibodies is required to synergistically target the IL6/JAK/STAT3 pathway and be used in the accurate treatment of COVID-19 infection. The use of the theranostics approach associated with COVID-19 treatment has improved a new generation of more selective nanosystems. The trend suggests that nanomedicine will be extensively used in the clinic in the near future, improving the population's quality of life.

\section{Funding}

This research received no external funding.

\section{Acknowledgments}

This research has no acknowledgment,

\section{Conflicts of Interest}

The authors declare no conflict of interest.

\section{References}

1. Cascella, M.; Rajnik, M.; Cuomo, A.; Dulebohn, S.C.; Di Napoli, R. Features, evaluation and treatment coronavirus (COVID-19). Statpearls [internet]. 2020, StatPearls Publishing.

2. Xu, Z.; Shi, L.; Wang, Y.; Zhang, J.; Huang, L.; Zhang, C.; et al. Pathological findings of COVID-19 associated with acute respiratory distress syndrome. The Lancet respiratory medicine 2020, 8, 420-422, https://doi.org/10.1016/S2213-2600(20)30076-X.

3. Wang, Ting; Lund, Brady. Announcement information provided by United States' public libraries during the 2020 COVID-19 pandemic. Public Library Quarterly, 2020, 39.4: 283-294.

4. Kim, J.M.; Chung, Y.S.; Jo, H.J.; Lee, N.J.; Kim, M.S.; Woo, S.H.; et al. Identification of Coronavirus Isolated from a Patient in Korea with COVID-19. Osong Public Health and Research Perspectives 2020, 11, 3, https://doi.org/10.24171/j.phrp.2020.11.1.02.

5. Chen, Y.; Liu, Q.; Guo, D. Emerging coronaviruses: genome structure, replication, and pathogenesis. J Med Virol 2020, 92, 418-423, https://doi.org/10.1002/jmv.25681.

6. Walls, A.C.; Tortorici, M.A.; Snijder, J.; Xiong, X.; Bosch, B.J.; Rey, F.A.; Veesler, D. Tectonic conformational changes of a coronavirus spike glycoprotein promote membrane fusion. Proceedings of the National Academy of Sciences 2017, 114, 11157-11162, https://doi.org/10.1073/pnas.1708727114.

7. Park, J.E.; Li, K.; Barlan, A.; Fehr, A.R.; Perlman, S.; McCray, P.B.; Gallagher, T. Proteolytic processing of Middle East respiratory syndrome coronavirus spikes expands virus tropism. Proc Natl Acad Sci 2016, 113, 12262-12267, https://doi.org/10.1073/pnas.1608147113.

8. Walls, A.C; Park, Y.J.; Tortorici, M.A.; Wall, A.; McGuire, A.T.; Veesler, D. Structure, function, and antigenicity of the SARS-CoV-2 spike glycoprotein. Cell 2020, 181, 281-292, https://doi.org/10.1016/j.cell.2020.02.058.

9. Heurich, A.; Hofmann-Winkler, H.; Gierer, S.; Liepold, T.; Jahn, O.; Pöhlmann, S. TMPRSS2 and ADAM17 cleave ACE2 differentially and only proteolysis by TMPRSS2 augments entry driven by the severe acute respiratory syndrome coronavirus spike protein. $J$ Virol 2014, 88, 1293-1307, https://doi.org/10.1128/JVI.02202-13. 
10. Hoffmann, M.; Kleine-Weber, H.; Schroeder, S.; Krüger, N.; Herrler, T.; Erichsen, S. et al. SARS-CoV-2 cell entry depends on ACE2 and TMPRSS2 and is blocked by a clinically proven protease inhibitor. Cell 2020. 181, 271-280, https://doi.org/10.1016/j.cell.2020.02.052.

11. Zhu, X.; Liu, Q.; Du, L.; Lu, L.; Jiang, S. Receptor-binding domain as a target for developing SARS vaccines. J Thorac Dis 2013, 5, S142-8, https://doi.org/10.3978/j.issn.2072-1439.2013.06.06.

12. Conti, P.; Ronconi, G.; Caraffa, A.; Gallenga, C.E.; Ross, R.; Frydas, I.; Kritas, S.K. Induction of proinflammatory cytokines (IL-1 and IL-6) and lung inflammation by Coronavirus-19 (COVI-19 or SARS-CoV2): anti-inflammatory strategies.J Biol Regul Homeost Agents 2020, 34, 327-331, https://doi.org/10.23812/CONTI-E.

13. Kaplanski, G.; Marin, V.; Montero-Julian, F.; Mantovani, A.; Farnarier, C. IL-6: a regulator of the transition from neutrophil to monocyte recruitment during inflammation. Trends Immunol 2003, 24, 25-29, https://doi.org/10.1016/S1471-4906(02)00013-3.

14. Kaur, S.; Bansal, Y.; Kumar, R.; Bansal, G. A panoramic review of IL-6: Structure, pathophysiological roles $\begin{array}{llllll}\text { and inhibitors. Bioorganic } \quad \& \quad \text { Medicinal } & \text { Chemistry } & \text { 2020, } & 115327,\end{array}$ https://doi.org/10.1016/j.bmc.2020.115327.

15. Schaper, F.; Rose-John, S. Interleukin-6: biology, signaling and strategies of blockade. Cytokine Growth Factor Rev 2015, 26, 475-487, https://doi.org/10.1016/j.cytogfr.2015.07.004.

16. Hirano, T.; Yasukawa, K.; Harada, H.; Taga, T.; Watanabe, Y.; Matsuda, T.; et al. Complementary DNA for a novel human interleukin (BSF-2) that induces B lymphocytes to produce immunoglobulin. Nature 1986, 324, 73-76, https://doi.org/10.1038/324073a0.

17. Ol, K.K.; Agachan, B.; Gormus, U.; Toptas, B.; Isbir, T. Cox-2 gene polymorphism and IL-6 levels in coronary artery disease. Genet Mol Res. 2011, 10, 810-816, https://doi.org/10.4238/vol10-2gmr967.

18. Johnson, D.E.; O'Keefe, R.A.; Grandis, J.R. Targeting the IL-6/JAK/STAT3 signalling axis in cancer. Nat Rev Clin Oncol 2018, 15, 234-248, https://doi.org/10.1038/nrclinonc.2018.8.

19. Narazaki, M.; Kishimoto, T. The two-faced cytokine IL-6 in host defense and diseases. Int J Mol Sci 2018, 19, 3528, https://doi.org/10.3390/ijms19113528.

20. Jones, S.A.; Jenkins, B.J. Recent insights into targeting the IL-6 cytokine family in inflammatory diseases and cancer. Nat Rev Immunol 2018, 18, 773-789, https://doi.org/10.1038/s41577-018-0066-7.

21. Iwamoto, N.; Yonezawa, A.; Matsubara, K.; Shimada, T. Acceleration of nano-surface and molecularorientation limited (nSMOL) proteolysis with acidified reduction pretreatment for quantification of Tocilizumab. Journal of pharmaceutical and biomedical analysis 2019, 164, 467-474, https://doi.org/10.1016/j.jpba.2018.11.019.

22. Garbers, C.; Heink, S.; Korn, T.; Rose-John, S. Interleukin-6: designing specific therapeutics for a complex cytokine. Nat Rev Drug Discov 2018, 17, 395-412, https://doi.org/10.1038/nrd.2018.45.

23. Zinzalla, G.; Haque, M.R.; Basu, B.P.; Anderson, J.; Kaye, S.L.; Haider, S.; et al. A novel small-molecule inhibitor of IL-6 signalling. Bioorganic \& medicinal chemistry letters 2010, 20, 7029-7032, https://doi.org/10.1016/j.bmcl.2010.09.117.

24. Singh, S., Gajulapati, V.; Gajulapati, K.; Goo, J.I.; Park, Y.H.; Jung, H.Y.; et al. Structure-activity relationship study of a series of novel oxazolidinone derivatives as IL-6 signaling blockers. Bioorg Med Chem Lett 2016, 26, 1282-1286, https://doi.org/10.1016/j.bmcl.2016.01.016.

25. Guo, J.; Yu, W.; Cai, G.; Zhang, W.; Li, S.; Zhu, J.; et al. Discovery of new benzensulfonamide derivatives as tripedal STAT3 inhibitors. Eur J Medl Chem 2018, 151, 752-764, https://doi.org/10.1016/j.ejmech.2018.03.053.

26. Zhang, W.; Zhao, Y.; Zhang, F.; Wang, Q.; Li, T.; Liu, Z.; et al. The use of anti-inflammatory drugs in the treatment of people with severe coronavirus disease 2019 (COVID-19): The experience of clinical immunologists from China. Clin Immunol 2020, 108393, https://doi.org/10.1016/j.clim.2020.108393.

27. Richardson, P.; Griffin, I.; Tucker, C.; Smith, D.; Oechsle, O.; Phelan, A.; Stebbing, J. Baricitinib as potential treatment for 2019-nCoV acute respiratory disease. Lancet 2020, 395, e30-e31, https://doi.org/10.1016/S0140-6736(20)30304-4.

28. Silva, G.A.; Ducheyne, P.; Reis, R.L. Materials in particulate form for tissue engineering. 1. Basic concepts. $J$ Tissue Eng Regen Med 2007, 1, 4-24, https://doi.org/10.1002/term.2.

29. Mazur, J.; Roy, K.; Kanwar, J.R. Recent advances in nanomedicine and survivin targeting in brain cancers. Nanomedicine 2018, 13, 105-137, https://doi.org/10.2217/nnm-2017-0286.

30. Lutz, J.F.; Lehn, J.M.; Meijer, E.W.; Matyjaszewski, K. From precision polymers to complex materials and systems. Nature Reviews Materials 2016, 1(5), 1-14, https://doi.org/10.1038/natrevmats.2016.24. 
31. Donskyi, I.; Drüke, M.; Silberreis, K.; Lauster, D.; Ludwig, K.; Kühne, C.; et al. Interactions of FullerenePolyglycerol Sulfates at Viral and Cellular Interfaces. Small 2018, 14, 1800189, https://doi.org/10.1002/smll.201800189.

32. Baek, S.; Singh, R.K.; Khanal, D.; Patel, K.D.; Lee, E.J.; Leong, K.W.; et al. Smart multifunctional drug delivery towards anticancer therapy harmonized in mesoporous nanoparticles. Nanoscale 2015, 7, 1419114216, https://doi.org/10.1039/C5NR02730F.

33. Ulbrich, K.; Hola, K.; Subr, V.; Bakandritsos, A.; Tucek, J.; Zboril, R. Targeted drug delivery with polymers and magnetic nanoparticles: covalent and noncovalent approaches, release control, and clinical studies. Chem Rev 2016, 116, 5338-5431, https://doi.org/10.1021/acs.chemrev.5b00589.

34. Giulbudagian, M.; Hönzke, S.; Bergueiro, J.; Işı1k, D.; Schumacher, F.; Saeidpour, S.; et al. Enhanced topical delivery of dexamethasone by $\beta$-cyclodextrin decorated thermoresponsive nanogels. Nanoscale 2018, 10, 469-479, https://doi.org/10.1039/C7NR04480A.

35. Zhao, Z.; Harris, B.; Hu, Y.; Harmon, T.; Pentel, P.R.; Ehrich, M.; Zhang, C. Rational incorporation of molecular adjuvants into a hybrid nanoparticle-based nicotine vaccine for immunotherapy against nicotine addiction. Biomaterials 2018, 155, 165-175, https://doi.org/10.1016/j.biomaterials.2017.11.021.

36. Lee, H.; Lee, M.Y.; Bhang, S.H.; Kim, B.S.; Kim, Y.S.; Ju, J.H.; et al. Hyaluronate-gold nanoparticle/tocilizumab complex for the treatment of rheumatoid arthritis. Acs Nano 2014, 8, 4790-4798, https://doi.org/10.1021/nn500685h.

37. Li, G.; Fan, Y.; Lai, Y.; Han, T.; Li, Z.; Zhou, P.; et al. Coronavirus infections and immune responses. J Med Virol 2020, 92, 424-432, https://doi.org/10.1002/jmv.25685.

38. https://www.nibib.nih.gov/research/labs-at-nibib/laboratory-molecular-imaging-and-nanomedicine-lomint/theranostic (18 March 2021)

39. Picard, F.J.; Bergeron, M.G. Rapid molecular theranostics in infectious diseases. Drug Discov Today 2002, 7, 1092-1101, https://doi.org/10.1016/S1359-6446(02)02497-2.

40. Wu, T.; Tang, M. Review of the effects of manufactured nanoparticles on mammalian target organs. J Appl Toxicol 2018, 38, 25-40, https://doi.org/10.1002/jat.3499.

41. Hillyer, J.F.; Albrecht, R.M. Gastrointestinal persorption and tissue distribution of differently sized colloidal gold nanoparticles. J Pharm Sci 2001, 90, 1927-1936, https://doi.org/10.1002/jps.1143.

42. Tulinska, J.; Kazimirova, A.; Kuricova, M.; Barancokova, M.; Liskova, A.; Neubauerova, E.; et al. Immunotoxicity and genotoxicity testing of PLGA-PEO nanoparticles in human blood cell model. Nanotoxicology 2015, 9(sup1), 33-43, https://doi.org/10.3109/17435390.2013.816798.

43. Radomska, A.; Leszczyszyn, J.; Radomski, M.W. The nanopharmacology and nanotoxicology of nanomaterials: new opportunities and challenges. Adv in Clin Exp Med 2016, 25, 151-162. https://doi.org/10.17219/acem/60879.

44. Anselmo, A.C.; Mitragotri, S. Nanomaterials in the clinic: An update. Bioengineering \& Translational medicine 2016, 1, 10-29, https://doi.org/10.1002/btm2.10143.

45. Bejarano, J.; Navarro-Marquez, M.; Morales-Zavala, F.; Morales, J.O.; Garcia-Carvajal, I.; Araya-Fuentes, E.; et al. Nanoparticles for diagnosis and therapy of atherosclerosis and myocardial infarction: evolution toward prospective theranostic approaches. Theranostics 2018, 8, 4710-4732, https://doi.org/10.7150/thno.26284.

46. https://www.understandingnano.com/nanotechnology-medical-diagnosis.html (18 March 2021)

47. Aslan, B.; Ozpolat, B.; Sood, A.K.; Lopez-Berestein, G. Nanotechnology in cancer therapy. J Drug Target 2013, 21, 904-913, https://doi.org/10.3109/1061186X.2013.837469.

48. Petros, R.A.; DeSimone, J.M. Strategies in the design of nanoparticles for therapeutic applications. Nat Rev Drug Discov 2010, 9, 615-627, https://doi.org/10.1038/nrd2591.

49. Baetke, S.C.; Lammers, T.G.G.M.; Kiessling, F. Applications of nanoparticles for diagnosis and therapy of cancer. Br J Radiol 2015, 88, 20150207, https://doi.org/10.1259/bjr.20150207.

50. Atal, S.; Fatima, Z. IL-6 Inhibitors in the Treatment of Serious COVID-19: A Promising Therapy? Pharmaceutical Medicine 2020, 34, 223-231, https://doi.org/10.1007/s40290-020-00342-z.

51. Wong, X. Y.; Sena-Torralba, A.; Alvarez-Diduk, R.; Muthoosamy, K.; \& Merkoci, A. Nanomaterials for nanotheranostics: Tuning their properties according to disease needs. ACS Nano, 2020, 14, 2585-2627, https://doi.org/10.1021/acsnano.9b08133.

52. Sivasankarapillai, V. S.; Pillai, A. M.; Rahdar, A.; Sobha, A. P.; Das, S. S.; Mitropoulos, A. C.; et al. On facing the SARS-CoV-2 (COVID-19) with combination of nanomaterials and medicine: possible strategies and first challenges. Nanomaterials 2020, 10, 852, https://doi.org/10.3390/nano10050852. 
53. Liu, B.; Li, M.; Zhou, Z.; Guan, X.; Xiang, Y. Can we use interleukin-6 (IL-6) blockade for coronavirus disease 2019 (COVID-19)-induced cytokine release syndrome (CRS)?. J Autoimmun, 2020, 102452, https://doi.org/10.1016/j.jaut.2020.102452.

54. Luo, P.; Liu, Y.; Qiu, L.; Liu, X.; Liu, D.; Li, J. Tocilizumab treatment in COVID-19: a single center experience. J Med Virol 2020, 92, 814-818, https://doi.org/10.1002/jmv.25801.

55. Peters, E.B.; Kibbe, M.R. Nanomaterials to Resolve Atherosclerosis. ACS Biomater Sci Eng 2020, 6,7, 36933712, https://doi.org/10.1021/acsbiomaterials.0c00281.

56. Nikaeen, G.; Abbaszadeh, S.; Yousefinejad, S. Application of nanomaterials in treatment, anti-infection and detection of coronaviruses. Nanomedicine, 2020, 15, 1501-1512, https://doi.org/10.2217/nnm-2020-0117. 